\title{
FTCOT: A Fault Tolerant Transaction Commit Protocol with Timeout Constraint for Mobile Environment
}

\author{
Amit Malik ${ }^{1}$ and Muzammil Hasan ${ }^{2}$ \\ ${ }^{1}$ M.Tech Student, Department of Computer Science \& Engineering, Madan Mohan \\ Malviya University of Technology, Gorakhpur(U.P.),India \\ ${ }^{2}$ Assistant Professor, Department of Computer Science \& Engineering, Madan \\ Mohan Malviya University of Technology, Gorakhpur(U.P.),India \\ Email:amitrajeev@gmail.com ${ }^{1}$, muzammil.mmmec@gmail.com ${ }^{2}$
}

\begin{abstract}
Now day's transaction failure is a common issue in mobile environment. There are various cause of transaction failure in mobile environment, some of them most common are failure of coordinator (normally which is base station in case of mobile environment), disconnection of mobile device, low battery, handoff. This paper proposes the new transaction commit protocol, Fault Tolerant Transaction Commit Protocol with Timeout Constraint (FTCOT).It support coordinator failure, handoff (in case of mobility) which reduce transaction failure. This paper extends the feature of TCOT.
\end{abstract}

Keywords: Mobile Transaction, Handoff, Commit Set, Token

\section{Introduction}

The transaction is set of read \& write operation perform on database which transfer database from consistent state to another. A mobile transaction involve minimum of one mobile host, which initiate transaction, the transaction may not be completely executed on mobile host, so that transaction is fragmented and distributed among database server for execution, final result come back to same mobile unit. This create distributed mode of execution. When a transaction take place atomicity must be maintain, but maintaining atomicity in mobile environment more challenging task then fixed environment.

The challenges include network failure e.g. network partitioning and node disconnection, coordinator failure each of which involve risk of infinite blocking and can lead to higher number of aborts. With this mode of execution it is difficult to ensure ACID properties of transaction. Thus new model are being created for deal this environment and handle coordinator failure. To overcome from these issue various protocol like ICP, UCM, M2PC, TCOT have been designed for mobile environment. Integrated commit protocol for Mobile Network (ICP) is a protocol which handles coordinator failure or network partitioning but the main problem with this protocol in case of coordinator failure it transfers more number of messages as compare to other protocol. To be more reliable and efficient, we have designed a transaction commit protocol for mobile environment which handle coordinator failure in more convenient way as compare to other protocol. In this protocol for handle coordinator failure we use MSC (Part of fixed network). We use MSC for storing token (contain commit set, execution detail of transaction) which is created by coordinator at the time when transaction is initiated. 


\section{Proposed Model}

Fault Tolerant Transaction Commit Protocol with Timeout Constraints is a one phase transaction commit protocol, supports coordinator failure and mobility (handoff). It eliminates the voting phase of $2 \mathrm{PC}$ and an extension of TCOT. Fig 1 shows the architecture used with FTCOT consisting of participant (Mobile Host(MH), Fixed Host(FH)),Base Station (BS), Mobile Switching Centre(MSC), Coordinator(CO) where the CO is located on the base station, Fault Tolerant System (FTS) Where FTS is located on the MSC in fixed environment, participant database are also situated in fixed environment. A transaction is initiated from $\mathrm{MH}$, which fragment the transaction and calculate its timeout(Execution Timeout \& Shipping Timeout) for its part of transaction, then transfer remaining part of transaction to coordinator. Then coordinator executes the transaction and sends decision to $\mathrm{MH}$.

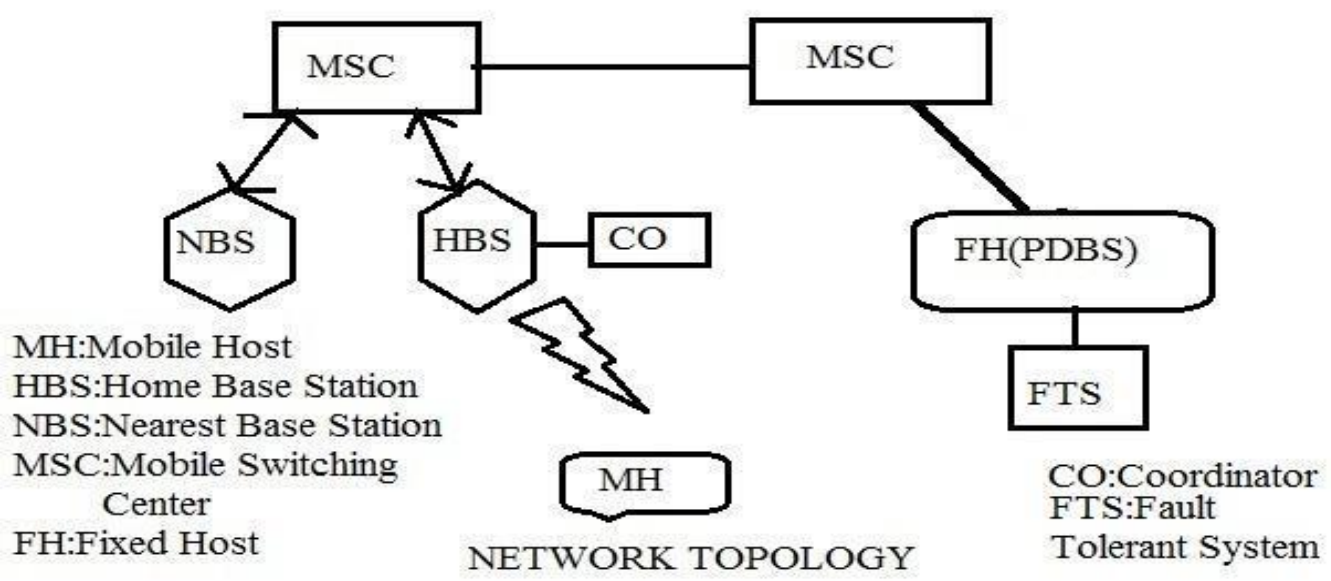

Figure 1. Typical Architecture for FTCOT

In this protocol we use two types of timeout: Execution Timeout and Update Shipping Timeout.

Execution Timeout (Et) defines an upper bound timeout value within which a node of a commit set completes the execution (not commit) of its ei. The value of Et may be node specific. It may depend on the size of ei and the characteristics of the processing unit. We identify H-MU's timeout by Et (MU ) and DBS timeout by Et(DBS). The relationship between these two timeouts is $\operatorname{Et}(\mathrm{MU})=\operatorname{Et}(\mathrm{DBS})+\Delta$ or $-\Delta$. The $\Delta$ accounts for the characteristics such as poor resources, disconnected state, availability of wireless channel, etc., compared to DBS. Furthermore, the value of a timeout for an ei depends on its MU, thus, Et (MUi) may not be equal to Et (MUj), ( $\mathrm{i}$ not equal to j). It is possible that a $\mathrm{MU}$ may take less time than its Et to execute its ei. We also do not rule out the possibility that in some cases Et(DBS) may be larger than Et (H-MU). Et typically should be just long enough to allow a fragment to successfully finish its entire execution in a normal environment (i.e., no failure of any kind, no message delay, etc.) 
Shipping timeout (St) defines the upper bound of the data shipping time from H-MU to DBS. Thus, at the end of Et the CO expects the updates to be shipped to the DBS and logged there within St. We compute St as Time to compose updates (Ut ) + Time for the updates to reach $\mathrm{CO}(\mathrm{Sh})$.

The FTCOT initiate and fragment transaction $\left(\mathrm{T}_{\mathrm{i}}\right)$ at $\mathrm{MH}$, the first fragment ei0 is being executed at $\mathrm{MH}$ and the remaining fragments of $\mathrm{Ti}$ i.e. $\mathrm{Ti}$-ei0 are sent to the Coordinator with execution timeout $\left(\mathrm{E}_{\mathrm{t}}\right) \&$ shipping timeout $\left(\mathrm{S}_{\mathrm{t}}\right), \mathrm{FTS}$ id to coordinator, then coordinator distribute transaction fragment to PDBS(PDBS) and create commit set(which contain all participants details ). After receiving their fragment participant calculate their execution timeout and send it to the coordinator after receiving all execution timeout coordinator create a token which contain commit set \& execution timeout to commit their part of fragment \& also shipping timeout. Now this token is sent to FTS which is used at the time of coordinator failure. At each time when any PDBS or MH extends it timeout then they send their extended timeout to coordinator the coordinator updates the token \& then said updated token to fts. After when PDBS or MH complete the processing of their part of fragments they send their decision to coordinator and then after receiving decision from all member of commit set coordinator finally execute the transaction.

\subsection{Algorithm For H-MU:-}

1. Initiate $\mathrm{T}_{\mathrm{i}}$

2.Set $\mathrm{H}-\mathrm{BS}$ as $\mathrm{CO}$, and store its ID

3. Set H-MSC as fault tolerant system

4. Extract its part of $e_{i}$ from $T_{i}$

5. Compute $\mathrm{E}_{\mathrm{t}} \& \mathrm{~S}_{\mathrm{t}}$ for its $\mathrm{e}_{\mathrm{i}}$

6. Send $E_{t}, S_{t}, T_{i}-e_{i}$ to $C O$

7. Start processing of its $e_{i}$

8. If extension of $E_{t}$ required

9. extend $\mathrm{E}_{\mathrm{t}}$ and $\mathrm{S}_{\mathrm{t}}$ and send to $\mathrm{CO}$

10 else

11. process $\mathrm{e}_{i}$

12. If H-MU want to Commit

13. then write update shipped to $\log$

14. update cached copy

15. Send updates to $\mathrm{CO}$ and start wait

16. If $\mathrm{CO}$ fails

17. then connect to new BS

18. sent FTS id to new BS

19. exchange(H-MU,new BS ID)

20. if abort message is received or $E_{t}, S_{t}$ expires

21. then write abort to $\log$

22. start abort procedure

23. else

24 .commit

\subsection{Algorithm for CO:-}

1. After receiving split T-e(MU), Create commit set

2. Send assignment to participant DBS 
3. Receive $\mathrm{E}_{\mathrm{t}}$ from all participant DBS

4. Create token write it to $\log$ and send to FTS

5. Wait for extension of $E_{t}$ and $S_{t}$ from participant

6. if any $E_{t}$ or $S_{t}$ is received

7. then update token

8. send extend $e_{t}$ or $S_{t}$ to FTS

9. If $\min \mathrm{E}_{\mathrm{t}}$ or $\mathrm{S}_{\mathrm{t}}$ expired or abort message is received

10. then write abort to $\log$

11. send global abort message to all participant

12. else

13. write commit to $\log$

14. commit

\subsection{Algorithm for Participant DBS:}

1. Receive assignment from $\mathrm{CO}$

2. Write assignment to $\log$

3. Compute $\mathrm{E}_{\mathrm{t}} \&$ send $\mathrm{E}_{\mathrm{t}}$ to $\mathrm{CO}$

4. Start processing of its $e_{i}$

5. If need of extension of $E_{t}$

6. then extend $E_{t}$

7. send it to $\mathrm{CO}$

8. If it receive global abort message

9. then write abort to $\log$

10. start abort procedure

11. If processing of its transaction complete

12.then send decision to $\mathrm{CO}$

13. If decision is commit

14.then write commit to $\log$

15. send commit to $\mathrm{CO}$

16.if decision is abort

17.then write abort to $\log$

18.send abort to $\mathrm{CO}$

\subsection{Algorithm For FTS(H-MSC):-}

1. Receive token from $\mathrm{CO}$

2. wait

3. If it receive any $E_{t}$ or $S_{t}$ from $C O$

4. then update tokent

5. if new BS ID and H-MU ID received then

6. sent token to New BS 


\subsection{Sequence Diagram: FTCOT}

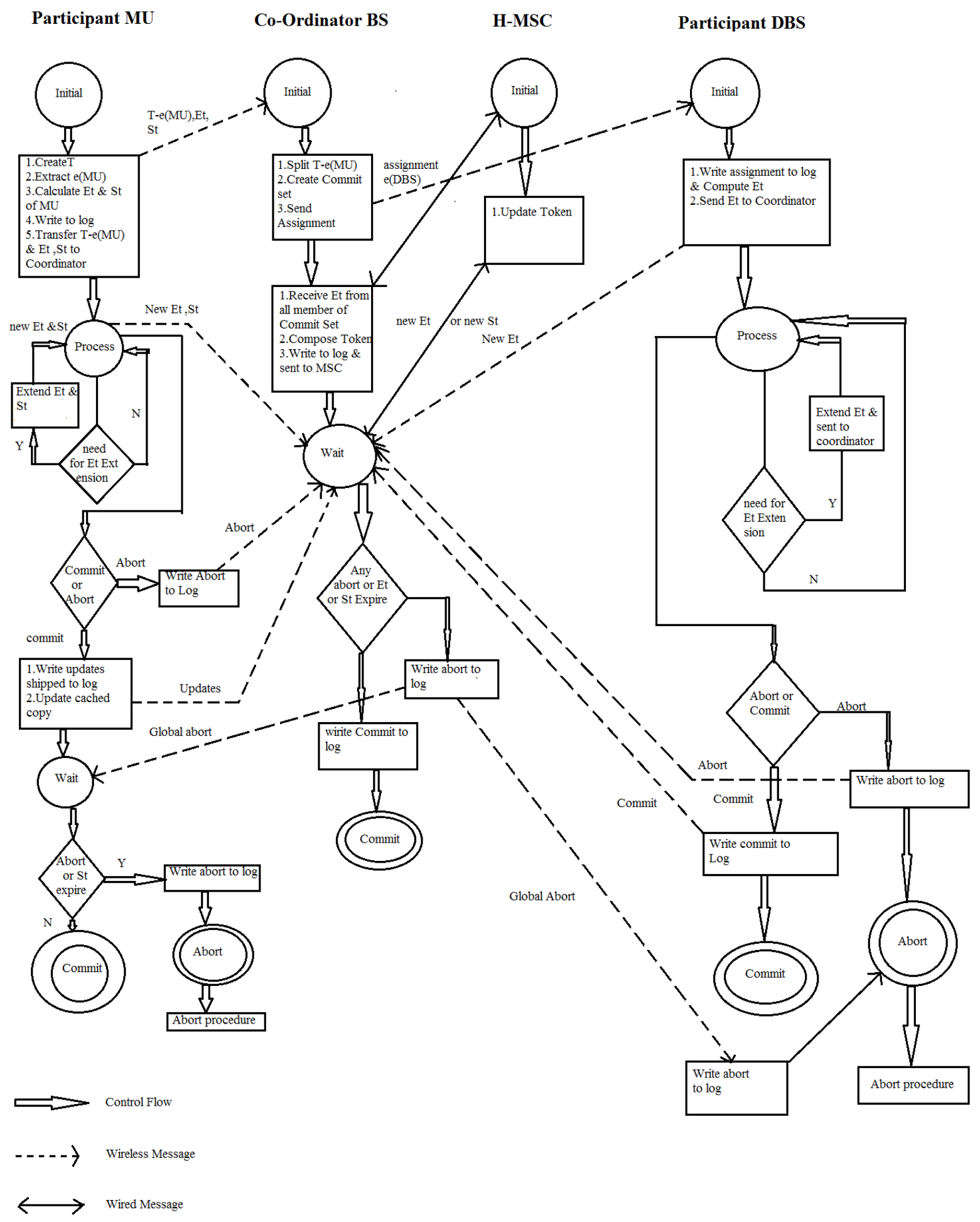

Figure 2. Sequence Diagram 


\subsection{Rule For increase $E_{t}$ (Execution Timeout) \& $S_{t}$ (Shipping Timeout):-}

1.If $\mathrm{H}-\mathrm{MU}$ want to increase $\mathrm{E}_{t}$ then it will increase $\mathrm{E}_{t}$ by only initial value of $\mathrm{E}_{t}$ only for 2 times and also increase $S_{t}$ by initial value of $E_{t}$.

2.If participant DBS want to increase its $E_{t}$ then it can only extend it by initial value of its $\mathrm{E}_{\mathrm{t}}$ only for 2 times.

\subsection{A Fault Tolerant:}

In case of failure of coordinator (H-BS) MH will connect to its nearest BS, which now act as a coordinator then new coordinator will communicate to MSC of previous BS and collect the token which was stored at MSC by failed coordinator, then it send token to MSC by updating it . The process of storing token at MSC introduced $\left(1+\mathrm{N}_{\text {ext }}\right)$ wired message in case of not any failure of coordinator.

If coordinator fails then 1 wireless message and 2 wired message are exchanged and total $4+\mathrm{N}_{\text {ext }}$ message are exchanged.

So it does not make any impact on commit time of our protocol because wired message does not make any impact on the performance of commit time of our protocol.

\subsection{B. Handoff:}

In case of handoff $\mathrm{MH}$ is got registered into nearest Base Station now which act as coordinator now in this case new base station exchange token with previous base station and send updated token to coordinator.

\subsection{Analysis of Message Transfer:}

As can be seen above by the sequence diagram number of wireless message transfer in an execution of transaction are when $\mathrm{CO}$ does not fail and handoff not occur

$2+\mathrm{N}_{\mathrm{ext}}$ (Where $\mathrm{N}_{\mathrm{ext}}$ is number of extension)

Or if we talk about the wired message introduce due to use of FTS for making system fault tolerant

$1+\mathrm{N}_{\mathrm{ext}}$ (If coordinator does not fail)

If Coordinator fails then number of wireless message and wired message transfer are

$3+\mathrm{N}_{\text {ext }}$ (wireless message)

$3+\mathrm{N}_{\text {ext }}$ (wired message)

In case of handoff then number of total message transfer in the successful execution of a transaction:

$3+\mathrm{N}_{\text {ext }}$ (wireless message)

$2+\mathrm{N}_{\mathrm{ext}}$ (wired message)

In case of mobile environment wireless message have impact on the performance of system while wired message does not affect the performance of system so here we concern only wireless message which are same as number of wireless message transfer in case of TCOT.

\section{Simulation}

The performance of Fault Tolerant Transaction Commit Protocol with Timeout Constraint detailed using simulation mode. The simulator is implemented in .NET platform using c\# language. It consist of Mobile Host, Which has Transaction Generator which generate transaction randomly, Transaction Fragment function which fragment transaction randomly. 
It consist a PDBS which executes its parts of transaction fragment and a coordinator which manage execution of transaction.

Transaction are generated by Transaction generator function at $\mathrm{MH}$, at each simulation run a parameter is used to define number of transaction to generate for simulation run. After that transaction is sent to transaction fragment function where transaction is fragmented. After that a fragment is assigned to $\mathrm{MH}$, on the basis of average time to perform read \& write operation on $\mathrm{MH}$, It calculate its execution timeout \& shipping timeout is calculated. After that remaining fragment transaction with $\mathrm{MH}$ timeout send to coordinator. Where coordinator assign fragment to PDBS, then PDBS calculate its timeout on the basis of average time to perform read \& write operation on the PDBS \& send it to coordinator. Then coordinator create a token which contain commit set \& execution timeout to execute each fragment, shipping timeout of MH After creating it send it to FTS. After that a function named parallel processing is called which further handle the execution of transaction.

A summary of the simulation parameter used in simulation model is presented by Table1.

Table 1. Simulation Parameters

\begin{tabular}{|l|l|l|}
\hline 01 & Mobile host & MH \\
\hline 01 & Participant database & PDBS \\
\hline 01 & Fault Tolerant System stored at MSC & FTS \\
\hline $40 \mathrm{~ms}$ & Average time to perform read operation at MH & T1 \\
\hline $60 \mathrm{~ms}$ & Average time to perform write operation at MH & T2 \\
\hline $30 \mathrm{~ms}$ & Average time to perform read operation at FH & T3 \\
\hline $50 \mathrm{~ms}$ & Average time to perform write operation at FH & T4 \\
\hline $50 \mathrm{~ms}$ & Average time to send updates to CO & T5 \\
\hline 2 & Max number of read operation transaction & Max1 \\
\hline 12 & & Max2 \\
\hline 1 & Max number of write operation in transaction & \\
\hline 6 & Minimum number of read operation in transaction & Min1 \\
\hline 02 & Minimum number of write operation in transaction & Min2 \\
\hline .010 & Number of transaction fragment & Fragmnet \\
\hline .005 & Fisconnection probability of MH & FailureProbMH \\
\hline .005 & Failure Probability of coordinator & FailureProbCO \\
\hline .02 & Probability of increase E $\mathrm{t}_{\mathrm{t}}$ by MH & FailureProbPDBS \\
\hline .02 & Probability of increase E $\mathrm{t}_{\mathrm{t}}$ by PDS & Prob1 \\
\hline 0 & Transaction generation time & Prob2 \\
\hline
\end{tabular}

\section{Result \&Discussion:}

\subsection{Number of Message abort due to Coordinator failure:}

When we perform simulation on the basis of above parameter, the simulation result shows that number of transaction abort in case of TCOT is more than FTCOT. Figure 3 shows that number of abort in case of TCOT more than FTCOT. 


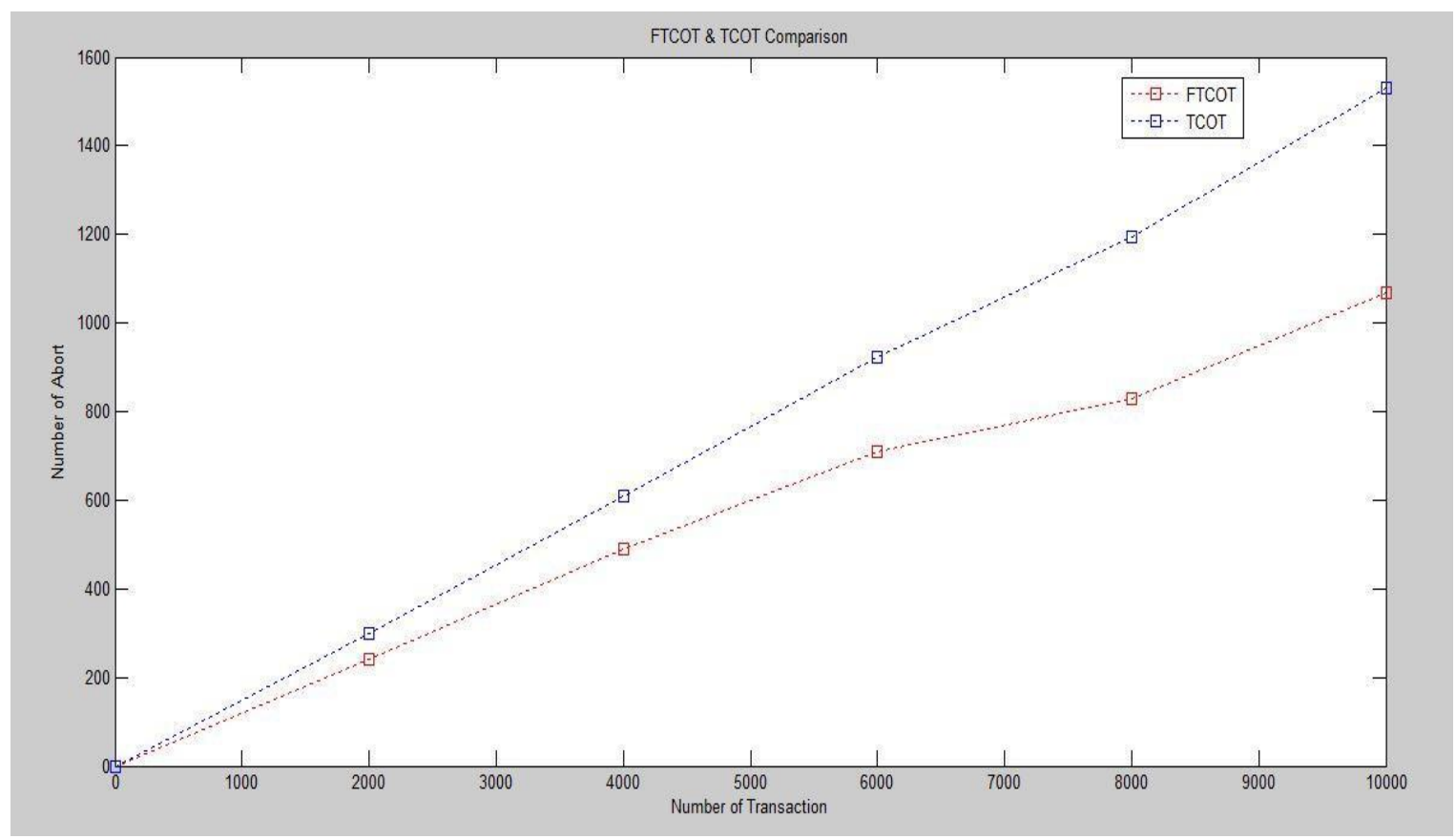

Figure 3. FTCOT \& TCOT Comparison

\section{B. Number of Wireless Message Transfer:}

Here we compare different protocol on the basis of number of wireless message transfer. We calculate the number of wireless message transfer for the M2PC,UCM \& FTCOT by assuming following parameter: Number of nodes taken place in execution is 2 . In case of FTCOT probability of Extension of timeout is 0.2 and probability of coordinator failure is 0.1 . It is clear from figure 4 number of wireless message transfer in case of FTCOT is less then M2PC \& UCM.

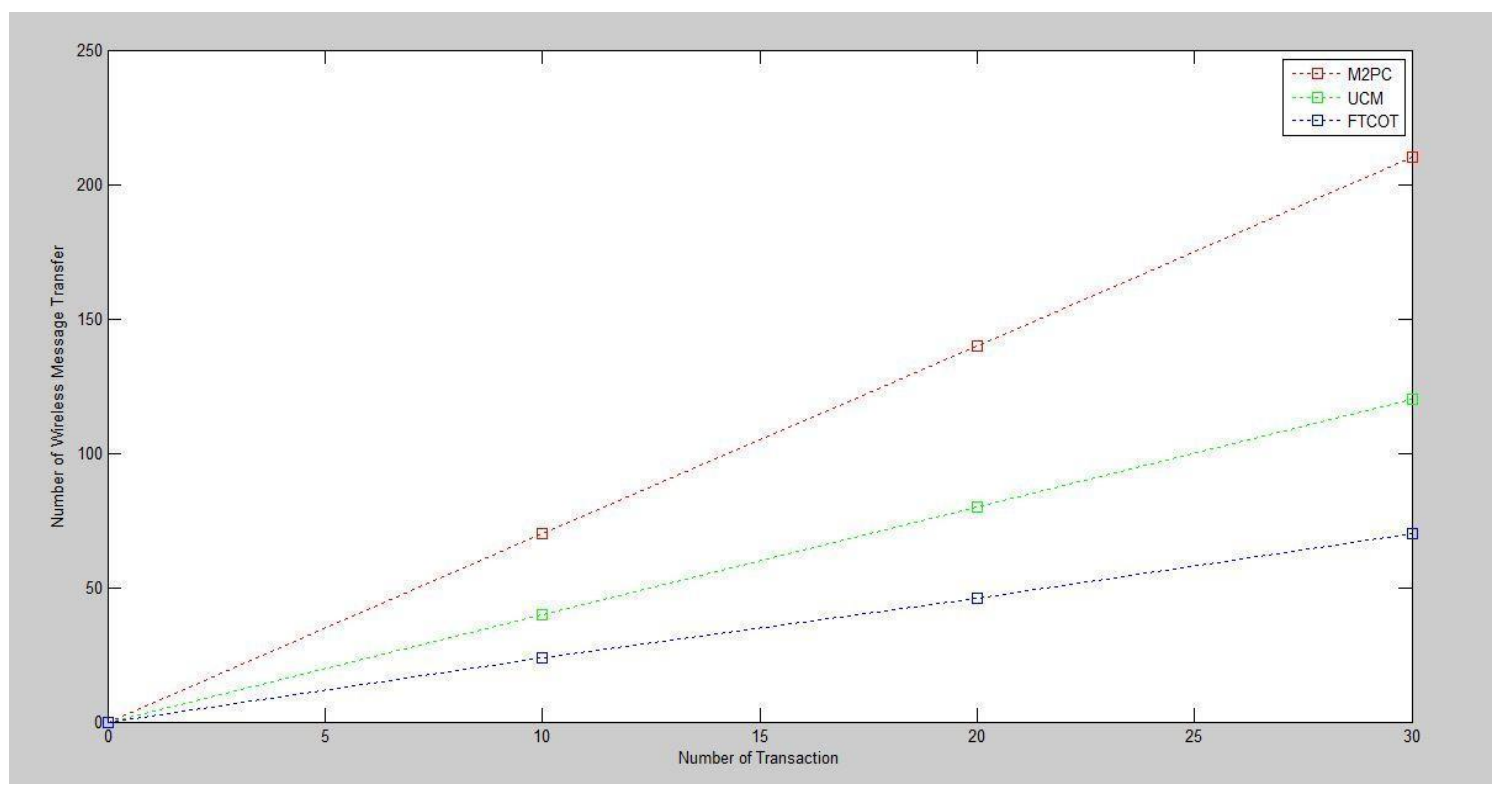

Figure 4. Number of Wireless Message Transfer 
Table 2. Number of Wireless Message Transfer

\begin{tabular}{|c|c|c|c|}
\hline $\begin{array}{c}\text { Number of } \\
\text { Wireless Message } \\
\text { Transfer/ Number } \\
\text { of Transaction }\end{array}$ & M2PC & UCM & FTCOT \\
\hline 10 & 70 & 40 & 24 \\
\hline 20 & 140 & 80 & 46 \\
\hline 30 & 210 & 120 & 76 \\
\hline
\end{tabular}

\section{Conclusion}

This paper presents an atomic commit protocol which is designed for mobile networking usage, because the traditional 2-Phase-Commit protocol is not suitable in mobile networks due to the blocking behavior in case of node or link failures .Our protocol is aimed at handling new challenges including Coordinator failure and Handoff. The FTCOT protocol avoids blocking situations; however it handles disconnection and mobility. Compared to the TCOT protocol, our protocol handle coordinator failure. The FTCOT protocol proposes an extension to the TCOT, where it is able to handle Coordinator failure and mobility. Therefore, we consider our approach to be a useful contribution for performing transactions in mobile environments with one phase, reduced message complexity and supports handoffs and disconnectionsin case of wireless link failures.

\section{References}

[1] Jim Gray, the Transaction Concepts, "Virtue and Limitation (June 1981), Seventh International Conference on Very Large Database,” Tandem Computers Incorporated, Sept 1981, TR 81.3.

[2] Christophe Bobineau, Philippe Pucheral, Maha Abdallah, "A Unilateral Commit Protocol for Mobile and Disconnected Computing," In Proceedings of ther $12^{\text {th }}$ International Conferenceon Parrallel and Distributed Computing System (PDCS), Las Vegas, USA, 2000.

[3] Joos-Hendrik Bose, Stefan Bottcher, Le Grunewald, "An Integrated Commit Protocol for Mobile Network Databases," $9^{\text {th }}$ international database engineering \& application symposium (IDEAS 05) in IEEE 2005.

[4] Vijay Kumar, Nitin Prabhu, Maggie Dunhamy, Ayse Yasemin Seydim, "TCOT: A Timout Based Mobile Transaction Commitment Protocol," IEEE Transactions on Computers, 51(10), 2002.

[5] Jim Gray and Leslie Lamport, "Consensus on Transaction Commit," CoRR, cs.DC/0408036, 2004.

[6] N.Nouali, A. Doucet, and H. Drias, "A two-phase commit protocol for mobile wireless environment," In H. E.Williams and G. Dobbie, editors,Sixteenth Australasian Database Conference (ADC2005), volume 39 of CRPIT, pages 135-144, Newcastle, Australia, 2005. ACS.

[7] B. Harsoor, S.Ramachandram, "Reliable Timeout Based Commit Protocol," Proceedings of $2^{\text {nd }}$ International Workshop on Trust Management in P2P Systems (IWTMP2PS-2010) CNSA-2010, Springer Verlag 2010, pp. 417-423.

[8] Bharati Harsoor, Dr. S. Ramachandram, "Modified Reliable Timeout base Commit Protocol," 978-1-457 70261-7/11, IEEE 2011.

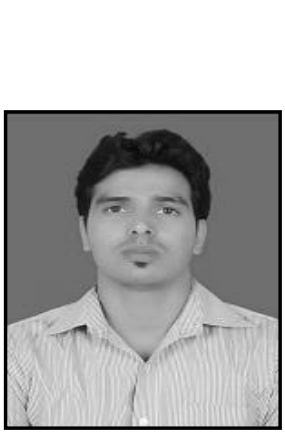

\section{Authors}

Amit Malik, obtained his bachelor degree in Information Technology from Anand Engineering College, Agra in 2010. Currently pursuing MTECH in Computer Science \& Engineering from Madan Mohan Malviya Engineering College Gorakhpur. His area of interest is Bio informatics, Database Transaction Model, Natural Language processing. 


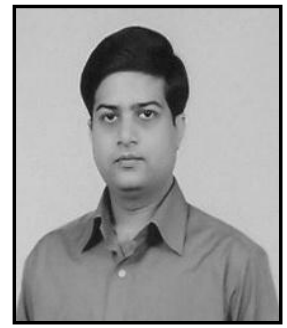

Mr Muzammil hasan, obtained his bachelor degree in Computer Science \& Engineering from Madan Mohan Malviya Engineering College in 2002, He also obtained his master degree in Computer Science \& Engineering From Madan Mohan Malviya Engineering College. He has 12 year teaching experience at UG \& PG level, Currently he is working as Assistant professor in MMMUT. His area of interest is real time database. 